Archive for

Organic Chemistry

Arkivoc 2020, part iv, 67-78

\title{
Hypervalent iodine-catalyzed conjugate addition with sulfonamides
}

\author{
Jeewani Poornima Ariyarathna, \& Elizabeth Claire Ziegelmeyer, ${ }^{\&}$ and Wei Li* \\ Department of Chemistry and Biochemistry, School of Green Chemistry and Engineering, The University of \\ Toledo, 2801 West Bancroft Street, Toledo, Ohio 43606, United States \\ Email: Wei.Li@Utoledo.edu
}

We dedicate this work in honor of Professor Viktor Zhdankin

Received 08-25-2020

Accepted 03-05-2021

Published on line 03-18-2021

\section{Abstract}

A hypervalent iodine-catalyzed conjugate addition with a sulfonamide nucleophile is described here. This reaction utilizes catalytic amounts of iodoarene and oxidant for the generation of the active hypervalent iodine catalyst. Activation of the enone substrate by the hypervalent iodine catalyst enables the nitrogen nucleophile addition that eventually forms a useful $\beta$-aminocarbonyl product. This hypervalent iodinecatalyzed Aza-Michael reaction represents an interesting alternative to existing conjugate addition protocols.
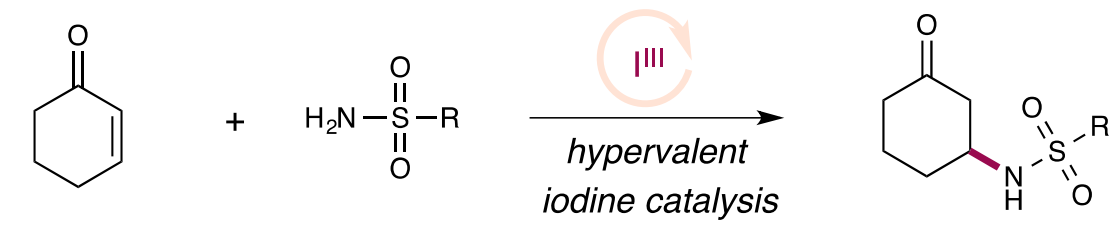

Keywords: hypervalent iodine catalysis, conjugate addition, $\beta$-aminocarbonyl, sulfonamide 


\section{Introduction}

Nitrogen-containing compounds are widely represented in pharmaceuticals, natural products, and agrochemicals. ${ }^{1}$ Consequently, a plethora of efforts have been devoted from the synthetic community focusing on accessing nitrogen-containing compounds. ${ }^{2-4}$ Among these endeavors, the aza-Michael addition reaction is an exceptional strategy to accessing $\beta$-aminocarbonyl compounds, nitrogen containing structures that can also serve as important building blocks to other useful ?-amino functional groups. ${ }^{5-8}$ Traditionally, the aza-Michael reactions are often promoted by strong acidic or basic conditions that are incompatible for substrates containing acid or base sensitive functional groups (Scheme 1a). ${ }^{9-11}$ Alternatively, a variety of transition metal and $\mathrm{Br} \varnothing \mathrm{nsted}$ catalysis including $\mathrm{Cu}, \mathrm{Ni}, \mathrm{Co}, \mathrm{Zr}, \mathrm{Al}, \mathrm{V}, p$-TsOH, etc., have been developed to circumvent the functional group intolerance issue. ${ }^{12-18}$ However, many of these catalyst systems are air and/or moisture sensitive, rendering their practicality somewhat limited. In addition, the presence of trace toxic metals raises concern in terms of their applications in medicinal synthesis. Therefore, a practical and metalfree catalytic aza-Michael protocol is still highly desired. ${ }^{19-22}$

a. aza-Michael reaction.

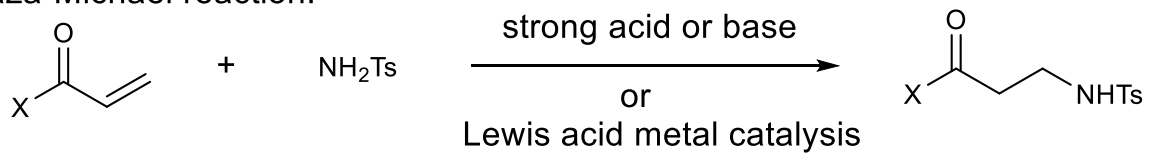

b. hypervalent iodine catalysis

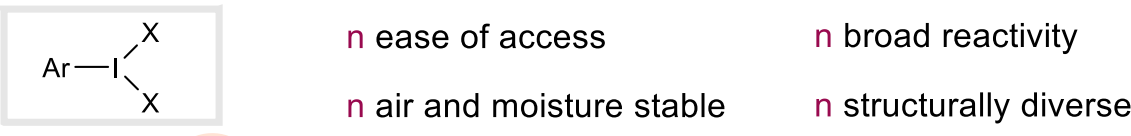

c. This work - $\left.\right|^{\prime \prime I}$

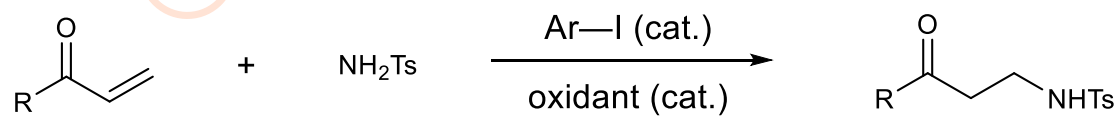

Scheme 1. Background.

The advent of hypervalent iodine compounds and their utilization in catalysis has been extensively documented in a number of reviews over the past several decades (Scheme 1b). ${ }^{23-25}$ In short, features including ease of access, air and moisture stability, structural diversity, and a broad range of reactivity renders hypervalent iodine reagents highly attractive in catalysis and organic synthesis. Hypervalent iodine catalysis has been extensively applied in a number of reaction settings such as oxidation, spirolactonization, rearrangement processes, alkene difunctionalizations, etc. ${ }^{26-32}$ This mode of catalysis has been restricted to electron-rich olefin structures due to the electrophilic nature of the hypervalent iodine catalyst. For electrondeficient olefins, this class of catalyst often proves to be rather challenging with a few exceptions. ${ }^{33-35}$ Given these features, we wondered if the practical features of hypervalent iodine catalyst could be suited for the development of an efficient aza-Michael reaction. As part of our research interest in synthetic methods design to access useful nitrogen-containing compounds, ${ }^{36-44}$ we disclose here a practical hypervalent iodine-catalyzed aza-Michael reaction with simple sulphonamides as the nitrogen nucleophile (Scheme 1c). 


\section{Results and Discussion}

Our work started with cyclohexenone $\mathbf{1}$ and sulfonamide $\mathbf{2}$ as the model substrates. In terms of the hypervalent iodine catalyst, we adopted the use of iodotoluene as the catalyst precursor and selectfluor as the oxidant since such a protocol is well known in hypervalent iodine catalysis literature. ${ }^{33,34}$ Our initial solvent choice of dichloromethane (DCM), unfortunately, did not provide any desired product (Table 1, entry 1). Gratifyingly, the reaction provided the desired product in 53\% yield when we changed the solvent to acetonitrile (MeCN) (Table 1, entry 2). Following this lead, we screened several catalyst and oxidant loading combinations, however, the reaction yield did not improve drastically and stayed consistent around $50 \%$ yield (Table 1, entry 3-5). In cases, the increase of oxidant loading seemed detrimental to the product yield (Table 1, entry 4). During our stoichiometry studies, we realized that a simple increase in the cyclohexenone loading to 2 equivalents afforded the most optimal reaction conditions (Table 1, entry 6 ). Furthermore, control reactions demonstrated that both the catalyst and the oxidant are essential to a productive reaction.

Table 1. Optimization studies

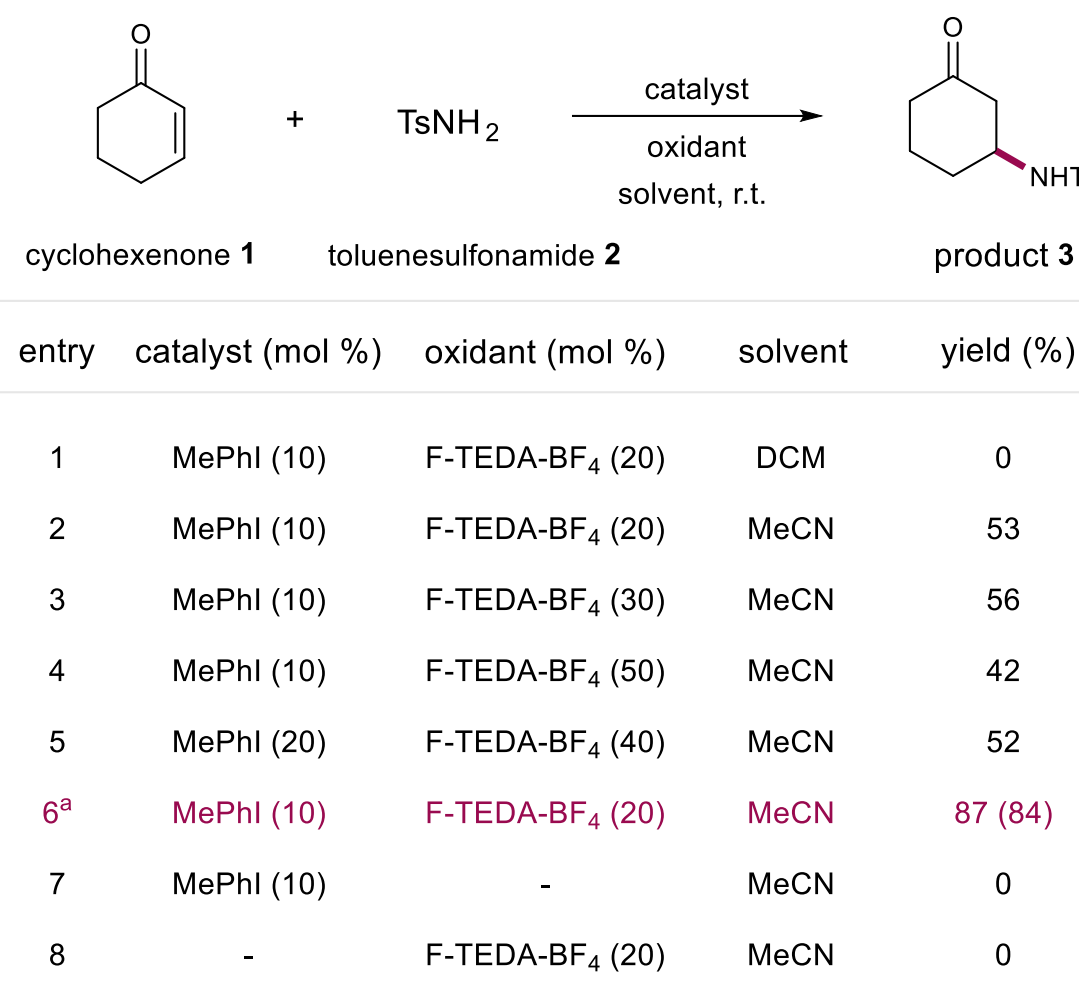

a. Standard conditions: $1(0.4 \mathrm{mmol}), 2(0.2 \mathrm{mmol})$, iodoarene catalyst $(0.02$ $\mathrm{mmol})$, oxidant $(0.04 \mathrm{mmol}), \operatorname{MeCN}(0.5 \mathrm{M})$, r.t., $16 \mathrm{~h}$. Yields were determined by ${ }^{1} \mathrm{H}$ NMR analysis using 1,3-benzodioxole as the internal standard. Yield in parenthesis was isolated yield.

With the optimized conditions in hand, we quickly evaluated a preliminary scope for this reaction. In this case, a number of sulfonamide substrates readily provided the desired products in good to great yields (Table 2). For instance, both electron-donating and-deficient substituents on the sulfonamides were well tolerated (Table 2, products 4-7). Sulfonamides containing heteroaromactics such as thiophenes and pyrazole, 
and cross-coupling ready functional group such as bromide were also viable substrates in this reaction (Table 2, products 8-10). In addition, alkyl sulfonamides including methyl, cyclopropyl, and camphor proceeded smoothly to afford the corresponding products (Table 2, products 11-13). In terms of the enone substrates, we demonstrated that cyclopentenone could also generate the desired conjugate addition product, albeit with diminishing efficiency (Table 2, product 14). To probe the utility of this reaction for pharmaceutical synthesis, we were delighted to show that the drug Celebrex could readily participate to afford the desired amination product 15 in 61\% yield (Table 2, product 15). These substrates, collectively, indicate that this conjugate addition protocol has the potential to be a widely applicable tool in the syntheses of $\beta$-aminocarbonyl motifs. Finally, we conducted derivatization studies using the standard product $\mathbf{3}$. In one case, we demonstrated a simple reduction of 3 with $\mathrm{LiAlH}_{4}$ and $\mathrm{AlCl}_{3}$ in $\mathrm{THF}$ to afford the product 16 in $69 \%$ yield. In another, we employed $\mathrm{MeMgBr}$ for a straightforward methyl addition for the ketone functionalization to observe the formation of product 17 in $62 \%$ yield (Scheme 2 ).

Table 2. Substrate scope studies

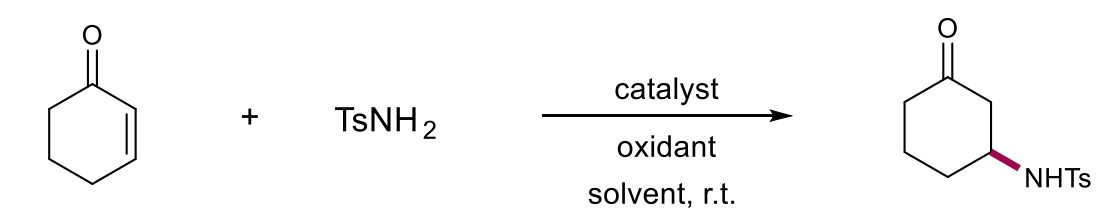

cyclohexenone 1 toluenesulfonamide 2

product 3<smiles>O=C1CCC[C@H](NS(=O)(=O)c2ccccc2)C1</smiles>

4, $77 \%$<smiles>CC(=O)c1ccccc1S(=O)(=O)N[C@H]1CCCC(=O)C1</smiles>

$7,61 \%$<smiles>Cc1nn(C)cc1S(=O)(=O)NC1CCCC(=O)C1</smiles>

$10,68 \%$<smiles>CC1(C)C2CCC1(CS(=O)(=O)NC1CCCC(=O)C1)C(=O)C2</smiles>

$13,47 \%, 1: 1 \mathrm{dr}$<smiles>COc1ccc(S(=O)(=O)NC2CCCC(=O)C2)cc1</smiles>

5, 64\%<smiles>O=C1CCC[C@H](NS(=O)(=O)c2cccs2)C1</smiles>

$8,73 \%$<smiles>CS(=O)(=O)N[C@H]1CCCC(=O)C1</smiles>

$11,63 \%$

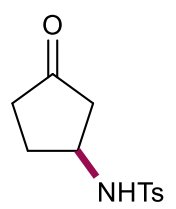

14, $39 \%$<smiles>O=C1CCC[C@H](NS(=O)(=O)c2ccc(OC(F)(F)F)cc2)C1</smiles>

6, $67 \%$<smiles>O=C1CCC[C@H](NS(=O)(=O)c2ccc(Br)s2)C1</smiles>

9, $81 \%$<smiles>O=C1CCC[C@H](NS(=O)(=O)C2CC2)C1</smiles>

$12,62 \%$<smiles>Cc1ccc(-c2cc(C)nn2-c2ccc(S(=O)(=O)N[C@H]3CCCC(=O)C3)cc2)cc1</smiles> 
<smiles>CNC1CCCC(O)C1</smiles>

16

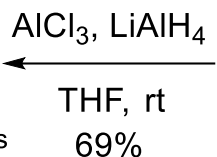

2.2:1 dr

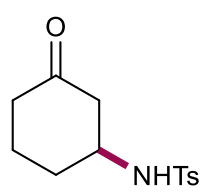

3

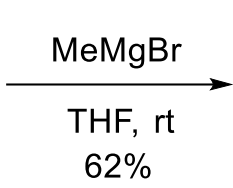

1.4:1 dr

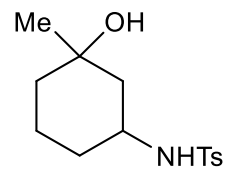

17

Scheme 2. Derivatization studies.

Having a reasonable substrate scope in hand and drawing inspiration from existing literatures of hypervalent iodine catalysis and conjugate addition reactions, we tentatively propose the following mechanistic framework for this reaction. ${ }^{45}$ To initiate the reaction, the iodoarene catalyst precursor is first oxidized to the active hypervalent iodine form $\mathbf{A}$, which is characterized and known from previous reports. ${ }^{46}$ The active catalyst then activates the $\alpha, \beta$-unsaturated ketone via the carbonyl oxygen coordination to generate $\mathbf{B}$. Conjugate addition by the sulfonamide nucleophile results in the enolate intermediate $\mathbf{C}$. Following this, proton transfer affords the conjugate addition product. During this step, the active catalyst is regenerated to undergo another cycle (Scheme 3).

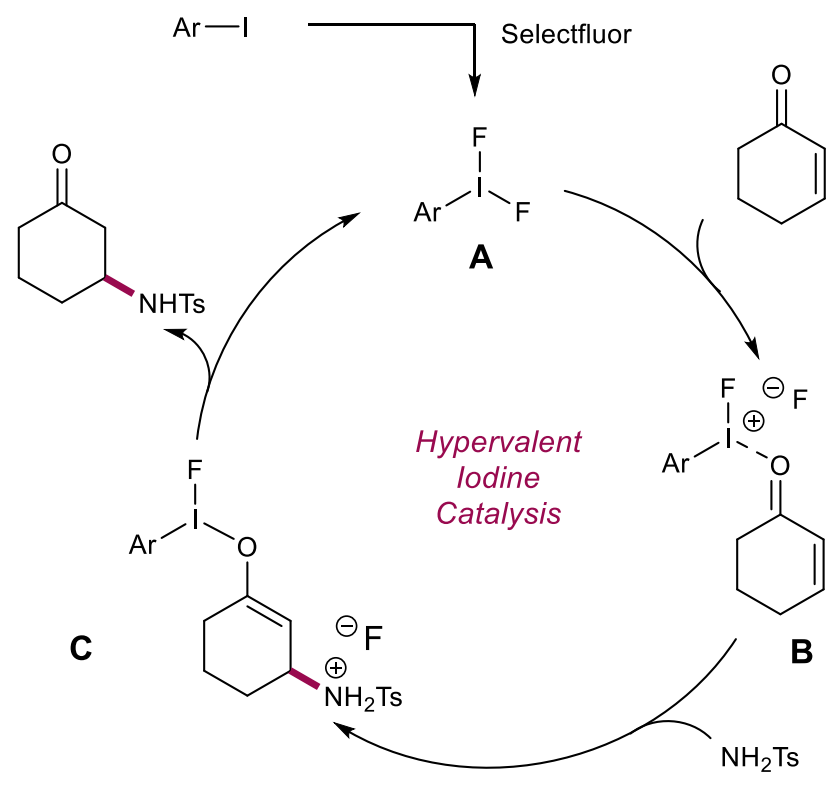

Scheme 3. Proposed catalytic cycle.

\section{Conclusions}

We have developed a useful protocol for the aza-Michael reaction using sulfonamides as the nitrogen nucleophile. This reaction tolerates a fairly extensive scope of sulfonamides including heteroaromatics, and in one case, even a drug molecule Celebrex can readily participate in this reaction. The practicality of this reaction is further testified with open-air conditions. We have also shown that the product can be easily 
derivatized into other useful ? ?-amino functionalities. Finally, our lab is currently in the process of developing an asymmetric variant of this reaction to further extend its synthetic utility.

\section{Experimental Section}

General. Commercial reagents and solvents were purchased from Sigma Aldrich, Oakwood Chemicals, Alfa Aesar, Matrix Scientific, Acros Organic and were used as received. Organic solutions were concentrated under reduced pressure on an IKA rotary evaporator using an acetone-dry ice bath. Chromatographic purification of products was accomplished using flash chromatography on 230-400 mesh silica gel. Thin-layer chromatography (TLC) was performed on Analtech $250 \mathrm{~mm}$ silica gel HLF UV-250 plates. Visualization of the developed plates was performed by fluorescent quenching and/or potassium permanganate. ${ }^{1} \mathrm{H}$ and ${ }^{13} \mathrm{C} N M R$ spectra were recorded on a Bruker instrument (600 and $150 \mathrm{MHz}$ ) or INOVA 600 (600 and $150 \mathrm{MHz}$ ) and are internally referenced to residual protio solvent signals (for $\mathrm{CDCl} 3,7.27$ and $77.0 \mathrm{ppm}$, respectively). Data for ${ }^{1} \mathrm{H}$ NMR are reported as follows: chemicals shift $(\mathrm{ppm})$, multiplicity $(\mathrm{s}=$ singlet, $\mathrm{d}=$ doublet, $\mathrm{t}=$ triplet, $\mathrm{q}=$ quartet, $\mathrm{h}=$ heptet, $\mathrm{m}=$ multiplet, $\mathrm{br}=$ broad), integration, coupling constant $(\mathrm{Hz}) \cdot{ }^{13} \mathrm{C}$ spectra were reported as chemical shifts in ppm and multiplicity where appropriate.

General Procedure for hypervalent iodine-catalyzed conjugate addition reaction. To an $8 \mathrm{~mL}$ vial equipped with a stir bar was added 4-iodotoluene $(5 \mathrm{mg}, 0.02 \mathrm{mmol}$ ), Selectfluor (14 $\mathrm{mg}, 0.04 \mathrm{mmol}$ ) and sulfonamide $(0.2 \mathrm{mmol})$. Then solvent ( $\mathrm{MeCN}, 0.4 \mathrm{~mL}$ ) was added via a syringe, followed by enone $(0.4 \mathrm{mmol})$. The reaction mixture was then stirred $16 \mathrm{~h}$ at room temperature. The reaction mixture was concentrated under reduced pressure to give the crude product, which was purified by column chromatography on silica gel to afford the pure product.

4-Methyl-N-(3-oxocyclohexyl)benzenesulfonamide (3). This compound was prepared according to the General Procedure using, 4-methylbenzenesulfonamide (34 mg, $0.2 \mathrm{mmol}$ ) and cyclohex-2-en-1-one (38 $\mu \mathrm{L}$, $0.4 \mathrm{mmol}$ ). After purification by column chromatography $\mathrm{SiO}_{2}$ (30\% to $50 \% \mathrm{EtOAc} / \mathrm{hexanes}$ ), The title compound was isolated as a white solid (45 mg, 84\% yield). ${ }^{1} \mathrm{H} \mathrm{NMR}\left(600 \mathrm{MHz}, \mathrm{CDCl}_{3}\right) \delta 7.75(\mathrm{~d}, J 9.0 \mathrm{~Hz}, 2 \mathrm{H})$, 7.30 (d, J 9.0 Hz, 2 H), 5.44 (d, J 9.0 Hz, 1 H), 3.56-3.46 (m, 1 H), 2.49 (dd, J 12.0, 4.5 Hz, 1 H), $2.43(\mathrm{~s}, 3 \mathrm{H}), 2.34-$ $2.26(\mathrm{~m}, 1 \mathrm{H}), 2.25-2.20(\mathrm{~m}, 2 \mathrm{H}), 2.02-1.91(\mathrm{~m}, 2 \mathrm{H}), 1.69-1.52(\mathrm{~m}, 2 \mathrm{H}) ;{ }^{13} \mathrm{C} \mathrm{NMR}\left(150 \mathrm{MHz}, \mathrm{CDCl}_{3}\right) \delta 208.2$, 143.7, 137.5, 129.8, 126.9, 52.4, 48.4, 40.5, 31.8, 21.6, 21.5; IR (Neat): 3225.2, 2953.4, 1700.7, 1451.6, 1326.3, $1150.11086 .5 \mathrm{~cm}^{-1}$. Melting point: $112-114{ }^{\circ} \mathrm{C}$. The spectra of this compound match previously reported literature. ${ }^{47}$

N-(3-oxocyclohexyl)benzenesulfonamide (4). This compound was prepared according to the General Procedure using, benzenesulfonamide $(31 \mathrm{mg}, 0.2 \mathrm{mmol}$ ) and cyclohex-2-en-1-one (38 $\mu \mathrm{L}, 0.4 \mathrm{mmol})$. After purification by column chromatography $\mathrm{SiO}_{2}$ (30\% to $50 \% \mathrm{EtOAc} /$ hexanes), The title compound was isolated as a white solid (39 mg, 77\% yield). ${ }^{1} \mathrm{H} N M R\left(600 \mathrm{MHz}, \mathrm{CDCl}_{3}\right) \delta 7.88(\mathrm{~d}, J 7.6 \mathrm{~Hz}, 2 \mathrm{H}), 7.60(\mathrm{t}, J 9.6 \mathrm{~Hz}, 1 \mathrm{H}), 7.53$ (t, J $7.7 \mathrm{~Hz}, 2 \mathrm{H}), 5.36(\mathrm{~d}, J 7.3 \mathrm{~Hz}, 1 \mathrm{H}), 3.61-3.52(\mathrm{~m}, 1 \mathrm{H}), 2.50(\mathrm{dd}, J$ 14.7, $4.8 \mathrm{~Hz}, 1 \mathrm{H}), 2.34-2.28(\mathrm{~m}, 1 \mathrm{H})$, 2.26-2.18 (m, $2 \mathrm{H}), 2.03-1.93(\mathrm{~m}, 2 \mathrm{H}), 1.70-1.55(\mathrm{~m}, 2 \mathrm{H}) ;{ }^{13} \mathrm{C} \mathrm{NMR}\left(150 \mathrm{MHz}, \mathrm{CDCl}_{3}\right) \delta 208.1,140.5,132.9$, 129.2, 126.8, 52.4, 48.4, 40.5, 31.8, 21.6; IR (Neat): 3256.2, 2946.4, 1709.7, 1452.7, 1321.5, $1152.6,1064.7 \mathrm{~cm}$ 1. Mp 110-112 ${ }^{\circ} \mathrm{C}$. HRMS (ESI) $\mathrm{m} / z$ calcd for $\mathrm{C}_{12} \mathrm{H}_{15} \mathrm{NNaO}_{3} \mathrm{~S}\left[(\mathrm{M}+\mathrm{Na})^{+}\right] 276.0670$, found 276.0667

4-Methoxy-N-(3-oxocyclohexyl)benzenesulfonamide (5). This compound was prepared according to the General Procedure using, 4-methoxybenzenesulfonamide (37 mg, $0.2 \mathrm{mmol}$ ) and cyclohex-2-en-1-one (38 $\mu \mathrm{L}$, $0.4 \mathrm{mmol}$ ). After purification by column chromatography $\mathrm{SiO}_{2}$ ( $30 \%$ to $50 \%$ EtOAc/hexanes), The title 
compound was isolated as a white solid (36 mg, 64\% yield). ${ }^{1} \mathrm{H} \mathrm{NMR}\left(600 \mathrm{MHz}, \mathrm{CDCl}_{3}\right) \delta 7.81(\mathrm{~d}, J 8.7 \mathrm{~Hz}, 2 \mathrm{H})$, $6.98(\mathrm{~m}, J 8.7 \mathrm{~Hz}, 2 \mathrm{H}), 5.18(\mathrm{~d}, J 7.3 \mathrm{~Hz}, 1 \mathrm{H}), 3.88(\mathrm{~s}, 3 \mathrm{H}), 3.56-3.47(\mathrm{~m}, 1 \mathrm{H}), 2.50(\mathrm{dd}, J 14.4,4.5 \mathrm{~Hz}, 1 \mathrm{H})$, 2.34-2.27 (m, $1 \mathrm{H}), 2.26-2.17(\mathrm{~m}, 2 \mathrm{H}), 2.03-1.92(\mathrm{~m}, 2 \mathrm{H}), 1.70-1.55(\mathrm{~m}, 2 \mathrm{H}) ;{ }^{13} \mathrm{C} \mathrm{NMR}\left(150 \mathrm{MHz}^{\mathrm{CDCl}}\right)_{3}$ $\delta$ 208.2, 163.0, 132.0, 129.1, 114.4, 55.6, 52.4, 48.4, 40.5, 31.8, 21.7; IR (Neat): 3183.0, 2960.1, 1698.5, 1594.2, $1498.9,1459.0,1258.9,1151.3,1089.9 \mathrm{~cm}^{-1}$. Mp 90-93 ${ }^{\circ} \mathrm{C}$. HRMS (ESI) $\mathrm{m} / z$ calcd for $\mathrm{C}_{13} \mathrm{H}_{17} \mathrm{NNaO}_{4} \mathrm{~S}\left[(\mathrm{M}+\mathrm{Na})^{+}\right]$ 306.0776, found 306.0774.

N-(3-oxocyclohexyl)-4-(trifluoromethoxy)benzenesulfonamide (6). This compound was prepared according to the General Procedure using, 4-(trifluoromethoxy)benzenesulfonamide (48 $\mathrm{mg}, 0.2 \mathrm{mmol}$ ) and cyclohex-2-en1-one ( $38 \mu \mathrm{L}, 0.4 \mathrm{mmol}$ ). After purification by column chromatography $\mathrm{SiO}_{2}$ ( $30 \%$ to $50 \% \mathrm{EtOAc} /$ hexanes), The title compound was isolated as a colorless oil (45 mg, 67\% yield). ${ }^{1} \mathrm{H} \mathrm{NMR}\left(600 \mathrm{MHz}, \mathrm{CDCl}_{3}\right) \delta 7.93(\mathrm{~d}, J 8.8 \mathrm{~Hz}$, $2 \mathrm{H}), 7.35$ (d, J $8.8 \mathrm{~Hz}, 2 \mathrm{H}), 5.47(\mathrm{~d}, J 7.8 \mathrm{~Hz}, 1 \mathrm{H}), 3.66-3.56(\mathrm{~m}, 1 \mathrm{H}), 2.55(\mathrm{dd}, J$ 14.4, $4.8 \mathrm{~Hz}, 1 \mathrm{H}), 2.37-2.30$ $(\mathrm{m}, 1 \mathrm{H}), 2.30-2.20(\mathrm{~m}, 2 \mathrm{H}), 2.05-1.94(\mathrm{~m}, 2 \mathrm{H}), 1.74-1.59(\mathrm{~m}, 2 \mathrm{H}) ;{ }^{13} \mathrm{C} \mathrm{NMR}\left(150 \mathrm{MHz}, \mathrm{CDCl}_{3}\right) \delta 208.2,152.2$, 138.9, 129.1, 121.0, 120.2 (q J $J_{C-F} 255 \mathrm{~Hz}$ ) , 52.6, 48.3, 40.5, 31.7, 21.5; IR (Neat): 3275.0, 2960.5, 1704.7, $1588.8,1491.8,1252.3,1207.6,1151.9,1090.1 \mathrm{~cm}^{-1}$. HRMS (ESI) $\mathrm{m} / z$ calcd for $\mathrm{C}_{13} \mathrm{H}_{14} \mathrm{~F}_{3} \mathrm{NNaO}_{4} \mathrm{~S}\left[(\mathrm{M}+\mathrm{Na})^{+}\right]$ 360.0493 , found 360.0491 .

Methyl 2-(N-(3-oxocyclohexyl)sulfamoyl)benzoate (7). This compound was prepared according to the General Procedure using, methyl 2-sulfamoylbenzoate ( $43 \mathrm{mg}, 0.2 \mathrm{mmol}$ ) and cyclohex-2-en-1-one (38 $\mu \mathrm{L}, 0.4 \mathrm{mmol}$ ). After purification by column chromatography $\mathrm{SiO}_{2}$ (30\% to $50 \%$ EtOAc/hexanes), The title compound was isolated as a colorless oil (38 mg, 61\% yield). ${ }^{1} \mathrm{H} \mathrm{NMR}\left(600 \mathrm{MHz}, \mathrm{CDCl}_{3}\right) \delta 8.13-8.08(\mathrm{~m}, 1 \mathrm{H}), 7.88-7.84(\mathrm{~m}, 1 \mathrm{H})$, 7.69-7.62 (m, $2 \mathrm{H}), 6.23(\mathrm{~d}, J 6.8 \mathrm{~Hz}, 1 \mathrm{H}), 3.98(\mathrm{~s}, 3 \mathrm{H}), 3.68-3.60(\mathrm{~m}, 1 \mathrm{H}), 2.48(\mathrm{dd}, J 14.4,4.5 \mathrm{~Hz}, 1 \mathrm{H}), 2.35-$ $2.28(\mathrm{~m}, 1 \mathrm{H}), 2.28-2.18(\mathrm{~m}, 2 \mathrm{H}), 2.07-1.95(\mathrm{~m}, 2 \mathrm{H}), 1.73-1.56(\mathrm{~m}, 2 \mathrm{H}) ;{ }^{13} \mathrm{C} \mathrm{NMR}\left(150 \mathrm{MHz}, \mathrm{CDCl}_{3}\right) \delta 207.8$, 168.1, 140.0, 132.5, 132.0, 131.0, 130.0, 129.4, 53.4, 52.9, 48.3, 40.5, 31.9, 21.7; IR (Neat): 3287.0, 2955.8, $1710.1,1435.0,1276.6,1161.0,1058.9 \mathrm{~cm}^{-1}$. HRMS (ESI) $\mathrm{m} / z$ calcd for $\mathrm{C}_{14} \mathrm{H}_{17} \mathrm{NNaO}_{5} \mathrm{~S}\left[(\mathrm{M}+\mathrm{Na})^{+}\right] 334.0725$, found 334.0723 .

$\mathbf{N}$-(3-Oxocyclohexyl)thiophene-2-sulfonamide (8). This compound was prepared according to the General Procedure using, thiophene-2-sulfonamide $(33 \mathrm{mg}, 0.2 \mathrm{mmol}$ ) and cyclohex-2-en-1-one (38 $\mu \mathrm{L}, 0.4 \mathrm{mmol}$ ). After purification by column chromatography $\mathrm{SiO}_{2}$ (30\% to $50 \%$ EtOAc/hexanes), The title compound was isolated as a white solid (38 mg, 73\% yield). ${ }^{1} \mathrm{H}$ NMR $\left(600 \mathrm{MHz}, \mathrm{CDCl}_{3}\right) \delta 7.62(\mathrm{dd}, J 12.9,4.8 \mathrm{~Hz}, 2 \mathrm{H}), 7.10(\mathrm{t}, J$ $4.3 \mathrm{~Hz}, 1 \mathrm{H}), 5.36$ (d, J $7.1 \mathrm{~Hz}, 1 \mathrm{H}), 3.70-3.60$ (m, $1 \mathrm{H}), 2.57$ (dd, J 14.2, $4.4 \mathrm{~Hz}, 1 \mathrm{H}), 2.37-2.31(\mathrm{~m}, 1 \mathrm{H}), 2.31-$ $2.21(\mathrm{~m}, 2 \mathrm{H}), 2.09-1.96(\mathrm{~m}, 2 \mathrm{H}), 1.75-1.59(\mathrm{~m}, 2 \mathrm{H}) ;{ }^{13} \mathrm{C} \mathrm{NMR}\left(150 \mathrm{MHz}, \mathrm{CDCl}_{3}\right) \delta 208.1,141.4,132.3,132.2$, 127.5, 52.8, 48.3, 40.5, 31.7, 21.6; IR (Neat): 3239.5, 3095.3, 2955.4, 1708.0, 1455.2, 1321.5, 1143.0, 1068.1 $\mathrm{cm}^{-1}$. Mp 113-115 ${ }^{\circ} \mathrm{C}$. HRMS (ESI) $\mathrm{m} / z$ calcd for $\mathrm{C}_{10} \mathrm{H}_{13} \mathrm{NNaO}_{3} \mathrm{~S}_{2}\left[(\mathrm{M}+\mathrm{Na})^{+}\right]$282.0235, found 282.0232.

5-Bromo- $\mathbf{N}$-(3-oxocyclohexyl)thiophene-2-sulfonamide (9). This compound was prepared according to the General Procedure using, 5-bromothiophene-2-sulfonamide (48 mg, $0.2 \mathrm{mmol}$ ) and cyclohex-2-en-1-one (38 $\mu \mathrm{L}, 0.4 \mathrm{mmol}$ ). After purification by column chromatography $\mathrm{SiO}_{2}$ ( $30 \%$ to $50 \% \mathrm{EtOAc} / \mathrm{hexanes}$ ), The title compound was isolated as a white solid (55 mg, 81\% yield). ${ }^{1} \mathrm{H} \mathrm{NMR}\left(600 \mathrm{MHz}, \mathrm{CDCl}_{3}\right) \delta 7.38(\mathrm{~d}, J 3.9 \mathrm{~Hz}, 1 \mathrm{H})$, $7.07(\mathrm{~d}, J 3.7 \mathrm{~Hz}, 1 \mathrm{H}), 5.28(\mathrm{~d}, J 7.1 \mathrm{~Hz}, 1 \mathrm{H}), 3.70-3.61(\mathrm{~m}, 1 \mathrm{H}), 2.62(\mathrm{dd}, J 14.4,4.8 \mathrm{~Hz}, 1 \mathrm{H}), 2.42-2.33(\mathrm{~m}, 1$ $\mathrm{H}), 2.32-2.23(\mathrm{~m}, 2 \mathrm{H}), 2.11-1.98(\mathrm{~m}, 2 \mathrm{H}), 1.77-1.64(\mathrm{~m}, 2 \mathrm{H}) ;{ }^{13} \mathrm{C} \mathrm{NMR}\left(150 \mathrm{MHz}, \mathrm{CDCl}_{3}\right) \delta 208.0,142.2,132.5$, 130.5, 120.2, 52.8, 48.3, 40.5, 31.8, 21.6; IR (Neat): 3156.2, 2958.2, 1690.8, 1465.8, 1405.6, 1326.3, 1152.8, $1075.0 \mathrm{~cm}^{-1}$. Mp 71-74 ${ }^{\circ} \mathrm{C}$. HRMS (ESI) $\mathrm{m} / z$ calcd for $\mathrm{C}_{10} \mathrm{H}_{12} \mathrm{BrNNaO}_{3} \mathrm{~S}_{2}\left[(\mathrm{M}+\mathrm{Na})^{+}\right]$359.9340, found 359.9337.

1,3-Dimethyl-N-(3-oxocyclohexyl)-1H-pyrazole-4-sulfonamide (10). This compound was prepared according to the General Procedure using, 1,3-dimethyl-1H-pyrazole-4-sulfonamide (35 mg, $0.2 \mathrm{mmol}$ ) and cyclohex-2en-1-one ( $38 \mu \mathrm{L}, 0.4 \mathrm{mmol})$. After purification by column chromatography $\mathrm{SiO}_{2}$ ( $70 \%$ to $100 \% \mathrm{EtOAc} /$ hexanes), The title compound was isolated as a white solid (37 mg, 68\% yield). $\left.{ }^{1} \mathrm{H} \mathrm{NMR} \mathrm{(600} \mathrm{MHz,} \mathrm{CDCl}_{3}\right) \delta 7.75(\mathrm{~s}, 1 \mathrm{H})$, 
4.67 (d, J $7.3 \mathrm{~Hz}, 1 \mathrm{H}), 3.87$ (s, $3 \mathrm{H}), 3.62-3.52(\mathrm{~m}, 1 \mathrm{H}), 2.61$ (dd, J 14.0, $4.5 \mathrm{~Hz}, 1 \mathrm{H}), 2.39(\mathrm{~s}, 3 \mathrm{H}), 2.37-2.32(\mathrm{~m}$, $1 \mathrm{H}), 2.30-2.21(\mathrm{~m}, 2 \mathrm{H}), 2.15-2.06(\mathrm{~m}, 1 \mathrm{H}), 2.05-1.97(\mathrm{~m}, 1 \mathrm{H}), 1.75-1.62(\mathrm{~m}, 2 \mathrm{H}) ;{ }^{13} \mathrm{C} \mathrm{NMR}(150 \mathrm{MHz}, \mathrm{CDCl} 3) \delta$ 207.9, 147.4, 134.0, 119.5, 52.3, 48.6, 40.6, 39.3, 32.1, 21.8, 12.3; IR (Neat): 3258.5, 3124.8, 2939.3, 1707.0, 1529.8, 1454.0, 1309.9, 1157.8, $1099.25 \mathrm{~cm}^{-1}$. Mp 120-122 ${ }^{\circ} \mathrm{C}$. HRMS (ESI) $\mathrm{m} / z$ calcd for $\mathrm{C}_{11} \mathrm{H}_{17} \mathrm{~N}_{3} \mathrm{NaO}_{3} \mathrm{~S}$ $\left[(\mathrm{M}+\mathrm{Na})^{+}\right] 294.0888$, found 294.0889 .

$\mathbf{N}$-(3-Oxocyclohexyl)methanesulfonamide (11). This compound was prepared according to the General Procedure using, methanesulfonamide (19 mg, $0.2 \mathrm{mmol}$ ) and cyclohex-2-en-1-one (38 $\mu \mathrm{L}, 0.4 \mathrm{mmol})$. After purification by column chromatography $\mathrm{SiO}_{2}$ (50\% to $70 \%$ EtOAc/hexanes), The title compound was isolated as a white solid (24 mg, 63\% yield). ${ }^{1} \mathrm{H}$ NMR (600 MHz, CDCl $) \delta 4.77(\mathrm{~d}, J 7.1 \mathrm{~Hz}, 1 \mathrm{H}), 3.85-3.77(\mathrm{~m}, 1 \mathrm{H}), 3.00(\mathrm{~s}$, $3 \mathrm{H}), 2.75$ (dd, J 14.4, $4.8 \mathrm{~Hz}, 1 \mathrm{H}), 2.43-2.35(\mathrm{~m}, 2 \mathrm{H}), 2.33-2.26(\mathrm{~m}, 1 \mathrm{H}), 2.22-2.15(\mathrm{~m}, 1 \mathrm{H}), 2.10-2.01(\mathrm{~m}, 1 \mathrm{H})$, 1.80-1.71 (m, $2 \mathrm{H}) ;{ }^{13} \mathrm{C}$ NMR (150 MHz, $\left.\mathrm{CDCl}_{3}\right) \delta$ 208.0, 52.7, 48.8, 42.1, 40.6, 32.2, 21.7; IR (Neat): 3259.5, 3007.8, 2955.8, 1703.4, 1451.0, 1306.4, 1143.9, $1082.6 \mathrm{~cm}^{-1}$. Mp 105-107 ${ }^{\circ} \mathrm{C}$. HRMS (ESI) m/z calcd for $\mathrm{C}_{7} \mathrm{H}_{13} \mathrm{NNaO}_{3} \mathrm{~S}\left[(\mathrm{M}+\mathrm{Na})^{+}\right] 214.0514$, found 214.0514 .

$\mathbf{N}$-(3-Oxocyclohexyl)cyclopropanesulfonamide (12). This compound was prepared according to the General Procedure using, cyclopropanesulfonamide $(24 \mathrm{mg}, 0.2 \mathrm{mmol})$ and cyclohex-2-en-1-one $(38 \mu \mathrm{L}, 0.4 \mathrm{mmol})$. After purification by column chromatography $\mathrm{SiO}_{2}$ (50\% to $70 \%$ EtOAc/hexanes), The title compound was isolated as a white solid (27 mg, $62 \%$ yield). ${ }^{1} \mathrm{H} \mathrm{NMR}\left(600 \mathrm{MHz}, \mathrm{CDCl}_{3}\right) \delta 4.80(\mathrm{~d}, J 7.8 \mathrm{~Hz}, 1 \mathrm{H}), 3.81-3.74(\mathrm{~m}, 1$ H), 2.77 (dd, J 14.4, 4.8 Hz $1 \mathrm{H}), 2.47-2.42(\mathrm{~m}, 1 \mathrm{H}), 2.41-2.32(\mathrm{~m}, 2 \mathrm{H}), 2.33-2.25(\mathrm{~m}, 1 \mathrm{H}), 2.24-2.17(\mathrm{~m}, 1 \mathrm{H})$, 2.08-2.01 (m, $1 \mathrm{H}), 1.80-1.67(\mathrm{~m}, 2 \mathrm{H}), 1.21-1.12(\mathrm{~m}, 2 \mathrm{H}), 1.08-0.98(\mathrm{~m}, 2 \mathrm{H}) ;{ }^{13} \mathrm{C} \mathrm{NMR}(150 \mathrm{MHz}, \mathrm{CDCl}) \delta$ 208.2, 52.6, 49.2, 40.6, 32.5, 31.4, 21.7, 5.9, 5.6; IR (Neat): 3243.6, 2958.9, 2883.2, 1706.2, 1455.7, 1317.6, 1138.8, 1149.8, $1071.1 \mathrm{~cm}^{-1}$. Mp 64-66 ${ }^{\circ} \mathrm{C}$. HRMS (ESI) $\mathrm{m} / z$ calcd for $\mathrm{C}_{9} \mathrm{H}_{15} \mathrm{NNaO}_{3} \mathrm{~S}\left[(\mathrm{M}+\mathrm{Na})^{+}\right] 240.0670$, found 240.0667.

1-(7,7-Dimethyl-2-oxobicyclo[2.2.1]heptan-1-yl)-N-(3-oxocyclohexyl)methanesulfonamide (13). This compound was prepared according to the General Procedure using, (1S)-10-Camphorsulfonamide (46 mg, 0.2 $\mathrm{mmol})$ and cyclohex-2-en-1-one $(38 \mu \mathrm{L}, 0.4 \mathrm{mmol})$. After purification by column chromatography $\mathrm{SiO}_{2}(50 \%$ to $70 \%$ EtOAc/hexanes), The title compound was isolated as a colorless oil and inseparable mixture of diastereomers (31 mg, 47\% yield). ${ }^{1} \mathrm{H}$ NMR $\left(600 \mathrm{MHz}, \mathrm{CDCl}_{3}\right) \delta 5.73\left(\mathrm{~d}, J 6.6 \mathrm{~Hz}, 1 \mathrm{H}_{\text {iso1 }}\right), 5.55(\mathrm{~d}, J 6.6 \mathrm{~Hz}, 1$ $\left.\mathrm{H}_{\text {iso2 }}\right), 3.89-3.77\left(\mathrm{~m}, 2 \mathrm{H}_{\text {iso1+iso2 }}\right), 3.40\left(\mathrm{~d}, J 15.1 \mathrm{~Hz}, 1 \mathrm{H}_{\text {iso1 }}\right), 3.37-3.33\left(\mathrm{~m}, 1 \mathrm{H}_{\text {iso2 }}\right), 3.01(\mathrm{~d}, J 10.5 \mathrm{~Hz}, 1 \mathrm{H}$ iso1) 2.98 (d, J $10.3 \mathrm{~Hz}, 1 \mathrm{H}$ iso2), 2.82 (dd, J 14.4, 4.8, Hz, $1 \mathrm{H}$ iso1), 2.71 (dd, J 14.4, 4.4 Hz, $1 \mathrm{H}$ iso2), 2.47-2.35 (m, $6 \mathrm{H}$ iso1+iso2), 2.34-2.26 (m, $2 \mathrm{H}$ iso1+iso2), 2.26-2.11 (m, $6 \mathrm{H}$ iso1+iso2), 2.11-1.98 (m, $5 \mathrm{H}$ iso1+iso2), 1.98-1.90 (m, $3 \mathrm{H}$ iso1+iso2), 1.86-1.68 (m, $4 \mathrm{H}_{\text {iso1+iso2) }}, 1.50-1.42\left(\mathrm{~m}, 2 \mathrm{H}_{\text {iso1+iso2 }),} 1.03\left(\mathrm{~s}, 3 \mathrm{H}_{\text {iso1 }}\right), 1.01\left(\mathrm{~s}, 3 \mathrm{H}_{\text {iso2 }}\right), 0.94\left(\mathrm{~s}, 3 \mathrm{H}_{\text {iso2 }}\right)\right.$, 0.92 (s, $3 \mathrm{H}_{\text {isol })}$ ); ${ }^{13} \mathrm{C}$ NMR $\left(150 \mathrm{MHz}, \mathrm{CDCl}_{3}\right) \delta 217.4,217.1,208.2,208.1,59.5,59.3,53.0,52.7,52.2,51.6,49.3$, 49.0, 48.8, 48.3, 43.0, 42.9, 42.8, 42.7, 40.7, 40.6, 32.7, 31.8, 27.1, 27.0, 27.0, 26.6, 21.8, 21.7, 20.0, 19.9, 19.5, 19.4; IR (Neat): 3279.8, 2956.2, 2892.0, 1737.1, 1709.0, 1450.0, 1418.5, 1320.4, 1143.7, $1052.4 \mathrm{~cm}^{-1}$. HRMS (ESI) $\mathrm{m} / \mathrm{z}$ calcd for $\mathrm{C}_{16} \mathrm{H}_{25} \mathrm{NNaO}_{4} \mathrm{~S}\left[(\mathrm{M}+\mathrm{Na})^{+}\right]$350.1402, found 350.1398.

4-Methyl-N-(3-oxocyclopentyl)benzenesulfonamide (14). This compound was prepared according to the General Procedure with slight modifications, using, 4-iodotoluene (10 mg, $0.04 \mathrm{mmol}$ ), Selectfluor (28 mg, 0.08 mmol) 4-methylbenzenesulfonamide (34 mg, $0.2 \mathrm{mmol}$ ) and cyclopent-2-en-1-one (33 $\mu \mathrm{L}, 0.4 \mathrm{mmol})$. After purification by column chromatography $\mathrm{SiO}_{2}$ (30\% to $50 \%$ EtOAc/hexanes), The title compound was isolated as a white solid (20 mg, 39\% yield). ${ }^{1} \mathrm{H}$ NMR $\left(600 \mathrm{MHz}, \mathrm{CDCl}_{3}\right) \delta 7.78(\mathrm{~d}, J 8.3 \mathrm{~Hz}, 2 \mathrm{H}), 7.33(\mathrm{~d}, J 8.3 \mathrm{~Hz}, 2 \mathrm{H}), 5.05$ $(\mathrm{d}, J 6.1 \mathrm{~Hz}, 1 \mathrm{H}), 3.91-3.83(\mathrm{~m}, 1 \mathrm{H}), 2.45(\mathrm{~s}, 3 \mathrm{H}), 2.41(\mathrm{dd}, J 18.7,7.0 \mathrm{~Hz}, 1 \mathrm{H}), 2.38-2.30(\mathrm{~m}, 1 \mathrm{H}), 2.28-2.19$ $(\mathrm{m}, 1 \mathrm{H}), 2.19-2.03(\mathrm{~m}, 2 \mathrm{H}), 1.91-1.83(\mathrm{~m}, 1 \mathrm{H}) ;{ }^{13} \mathrm{C} \mathrm{NMR}\left(150 \mathrm{MHz}, \mathrm{CDCl}_{3}\right) \delta 214.8,144.0,137.0,129.9,127.1$, 51.3, 45.3, 36.7, 30.2, 21.6; IR (neat): 3240.0, 3183.0, 2919.8, 1733.8, 1596.0, 1463.8, 1323.4, $1149.4,1091.3$ $\mathrm{cm}^{-1}$. Mp 108-110 ${ }^{\circ} \mathrm{C}$. HRMS (ESI) $\mathrm{m} / z$ calcd for $\mathrm{C}_{12} \mathrm{H}_{15} \mathrm{NNaO}_{3} \mathrm{~S}\left[(\mathrm{M}+\mathrm{Na})^{+}\right]$276.0670, found 276.0670 . 


\section{N-(3-Oxocyclohexyl)-4-(3-(trifluoromethyl)-5-(4-(trifluoromethyl)phenyl)-1H-pyrazol-1-}

yl)benzenesulfonamide (15). This compound was prepared according to the General Procedure using, Celecoxib (76 mg, $0.2 \mathrm{mmol}$ ) and cyclohex-2-en-1-one (38 $\mu \mathrm{L}, 0.4 \mathrm{mmol}$ ). After purification by column chromatography $\mathrm{SiO}_{2}$ (50\% to 70\% EtOAc/hexanes), The title compound was isolated as a white solid (65 mg, $61 \%$ yield). ${ }^{1} \mathrm{H}$ NMR (600 MHz, $\left.\mathrm{CDCl}_{3}\right) \delta 7.86(\mathrm{~d}, J 8.5 \mathrm{~Hz}, 2 \mathrm{H}), 7.49(\mathrm{~d}, J 8.5 \mathrm{~Hz}, 2 \mathrm{H}), 7.19(\mathrm{~d}, J 7.8 \mathrm{~Hz}, 2 \mathrm{H}), 7.11$ (d, J 8.1 Hz, 2 H), 6.76 (s, 1 H), 4.99 (d, J 7.3 Hz, 1 H), 3.65-3.57 (m, 1 H), 2.55 (dd, J 14.4, 4.8 Hz, 1 H), 2.39 (s, 3 $\mathrm{H}), 2.37-2.31(\mathrm{~m}, 1 \mathrm{H}), 2.29-2.18(\mathrm{~m}, 2 \mathrm{H}), 2.02-1.93(\mathrm{~m}, 2 \mathrm{H}), 1.71-1.60(\mathrm{~m}, 2 \mathrm{H}) ;{ }^{13} \mathrm{C} \mathrm{NMR}(150 \mathrm{MHz}, \mathrm{CDCl}) \delta$ 207.7, 145.3, $144.11\left(q, J_{C-F}=39.0 \mathrm{~Hz}\right), 142.6,140.0,139.8,129.8,128.7,127.9,125.7,125.6,121.0$ (q, JC-F $=$ $265.5 \mathrm{~Hz}$ ) 106.3, 52.6, 48.4, 40.5, 31.7, 21.6, 21.3; IR (Neat); 3266.0, 2955.4, 1708.0, 1596.6, 1471.4, 1235.6, 1158.1, 1131.1, $1094.4 \mathrm{~cm}^{-1}$. Mp 63-65 ${ }^{\circ} \mathrm{C}$. HRMS (ESI) $\mathrm{m} / \mathrm{z}$ calcd for $\mathrm{C}_{23} \mathrm{H}_{22} \mathrm{~F}_{3} \mathrm{~N}_{3} \mathrm{NaO}_{3} \mathrm{~S}\left[(\mathrm{M}+\mathrm{Na})^{+}\right] 500.1232$, found 500.1228 .

$\boldsymbol{N}$-(3-Hydroxycyclohexyl)-4-methylbenzenesulfonamide (16). To an $8 \mathrm{ml}$ vial equipped with magnetic stir bar was added 4-methyl- $\mathrm{N}$-(3-oxocyclohexyl)benzene sulfonamide $(53 \mathrm{mg}, 0.2 \mathrm{mmol}$ ) and anhydrous THF (1 mL). Then $\mathrm{AlCl}_{3}$ (40 mg, $0.3 \mathrm{mmol}$ ) was added, followed by $\mathrm{LiAlH}_{4}(12 \mathrm{mg}, 0.3 \mathrm{mmol}$ ). The reaction mixture was stirred at room temperature for $16 \mathrm{~h}$. Then the reaction was quenched with aqueous $\mathrm{NaOH}$ solution $(0.5 \mathrm{~mL}$, $1 \mathrm{M})$, and the organic layer was separated. The aqueous layer was extracted with EtOAc $(2 \times 2 \mathrm{~mL})$, and the combined organic layers were dried over anhydrous $\mathrm{Na}_{2} \mathrm{SO}_{4}$. The resulting solution was concentrated under reduced pressure. The crude product was isolated by column chromatography $\mathrm{SiO}_{2} \quad(30 \%$ to $50 \%$ EtOAc/hexanes), as a colorless oil (37 mg, 69\% yield). Only the major diastereomer is presented in the ${ }^{1} \mathrm{H}$ and ${ }^{13} \mathrm{C}$ NMR spectra. ${ }^{1} \mathrm{H}$ NMR $\left(600 \mathrm{MHz}, \mathrm{CDCl}_{3}\right) \delta 7.76(\mathrm{~d}, J 8.3 \mathrm{~Hz}, 2 \mathrm{H}), 7.30(\mathrm{~d}, J 8.1 \mathrm{~Hz}, 2 \mathrm{H}), 5.22$ (br. s., $\left.1 \mathrm{H}\right)$, 3.72 (br. s., $1 \mathrm{H}), 3.36-3.23(\mathrm{~m}, 1 \mathrm{H}), 2.43(\mathrm{~s}, 3 \mathrm{H}), 1.88(\mathrm{~d}, J 12.2 \mathrm{~Hz}, 1 \mathrm{H}), 1.81-1.72(\mathrm{~m}, 2 \mathrm{H}), 1.72-1.60(\mathrm{~m}, 2 \mathrm{H})$, 1.36-1.18 (m, $4 \mathrm{H}) ;{ }^{13} \mathrm{C}$ NMR $\left(150 \mathrm{MHz}, \mathrm{CDCl}_{3}\right) \delta 143.2,138.3,129.7,126.9,68.4,50.4,40.3$ 33.7, 32.5, 21.5, 19.2; IR; 3489.5, 3273.2, 2936.4, 2860.5, 1452.0, 1319.1, 1153.1, 1091.2, $1038.2 \mathrm{~cm}^{-1}$. HRMS (ESI) $\mathrm{m} / \mathrm{z}$ calcd for $\mathrm{C}_{13} \mathrm{H}_{19} \mathrm{NNaO}_{3} \mathrm{~S}\left[(\mathrm{M}+\mathrm{Na})^{+}\right]$292.0983, found 292.0984.

N-(3-hydroxy-3-methylcyclohexyl)-4-methylbenzenesulfonamide (17). To an $8 \mathrm{ml}$ vial equipped with magnetic stir bar was added 4-methyl- $\mathrm{N}$-(3-oxocyclohexyl) benzenesulfonamide (53 $\mathrm{mg}, 0.2 \mathrm{mmol}$ ) and anhydrous THF (1 mL). After cooling to $0{ }^{\circ} \mathrm{C}, \mathrm{MeMgBr}$ in THF $(1.7 \mathrm{~mL}, 0.5 \mathrm{mmol})$ was added. The reaction mixture was stirred at room temperature for $16 \mathrm{~h}$. Then the reaction was quenched with sat. $\mathrm{NH}_{4} \mathrm{Cl}$ solution (1 $\mathrm{mL})$, and the organic layer was separated. The aqueous layer was extracted with EtOAc $(2 \times 2 \mathrm{~mL})$, and the combined organic layers were dried over anhydrous $\mathrm{Na}_{2} \mathrm{SO}_{4}$. The resulting solution was concentrated under reduced pressure. The crude product isolated by column chromatography $\mathrm{SiO}_{2}$ ( $30 \%$ to $50 \%$ EtOAc/hexanes), as a colorless oil and inseparable mixture of diastereomers (35 mg, 62\% yield). ${ }^{1} \mathrm{H} \mathrm{NMR}\left(600 \mathrm{MHz}^{\mathrm{C}} \mathrm{CDCl}\right)_{3} \delta$

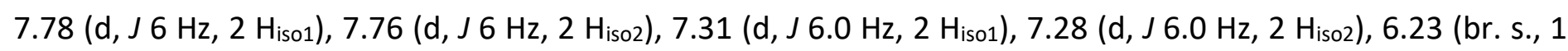

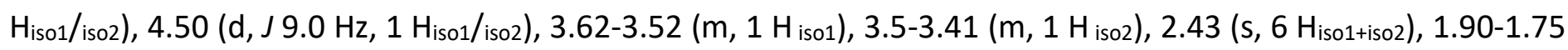

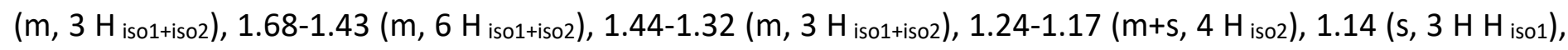
1.06-0.94 (m, $1 \mathrm{H} \mathrm{H}_{\text {isol }) ; ~}{ }^{13} \mathrm{C}$ NMR $\left(150 \mathrm{MHz} \mathrm{CDCl}_{3}\right) \delta 143.2,142.9,138.6,138.1,129.8,129.7,129.5,126.9$, 126.9, 71.2, 70.5, 49.6, 49.4, 46.4, 41.5, 38.6, 37.3, 33.3, 31.5, 31.3, 21.5, 21.5, 20.4, 17.0; IR; 3500.8, 3275.5, 2933.1, 2865.3, 1447.2, 1320.9, 1152.2, $1093.7 \mathrm{~cm}^{-1}$. HRMS (ESI) $\mathrm{m} / z$ calcd for $\mathrm{C}_{14} \mathrm{H}_{21} \mathrm{NNaO}_{3} \mathrm{~S}\left[(\mathrm{M}+\mathrm{Na})^{+}\right]$ 306.1140, found 306.1146 . 


\section{Acknowledgements}

\&These authors contributed equally to this manuscript.

\section{Supplementary Material}

The Supporting Information is available free of charge on the website.

\section{References}

1. Vitaku, E.; Smith, D. T.; Njardarson, J. T. Analysis of the Structural Diversity, Substitution Patterns, and Frequency of Nitrogen Heterocycles among U.S. FDA Approved Pharmaceuticals. J. Med. Chem. 2014, 57, 10257-10274.

https://doi.org/10.1021/jm501100b

2. Katritzky, A. R. Chem. Rev. 2004, 104, 2125-2126. https://doi.org/10.1021/cr0406413

3. Salat, K.; Moniczewski, A.; Librowski, T. Mini. Rev. Med. Chem. 2013, 13, 335-352. https://doi.org/10.2174/138955713804999801

4. Andrei, Y. Chem. Rev. 2014, 114, 7783-7783. https://doi.org/10.1021/cr500323m

5. Rulev, A. Y. Russ. Chem. Rev. 2011, 80, 197-218. https://doi.org/10.1070/RC2011v080n03ABEH004162

6. Sánchez-Roselló, M.; Aceña, J. L.; Simón-Fuentes, A.; del-Pozo, C. Chem. Soc. Rev. 2014, 43, 7430-7453. https://doi.org/10.1039/C4CS00156G

7. Shugrue, C. R. ; Featherston, A. L. ; Lackner, R. M. ; Lin, A. ; Miller, S. J. J. Org. Chem. 2018, 83, 4491-4504. https://doi.org/10.1021/acs.joc.8b00207

8. Romney, D. K. ; Colvin, S. M. ; Miller, S. J. J. Am. Chem. Soc. 2014, 136, 14019-14022. https://doi.org/10.1021/ja508757g

9. Lee, S.-J.; Ahn, J.-G.; Cho, C.-W.; Tetrahedron : Asymmetry 2014, 25, 1383-1388. https://doi.org/10.1016/j.tetasy.2014.09.002

10. Wang, J. ; Li, P.-F. ; Chan, S. H. ; Chan, A. S. C. ; Kwong, F. Y. Tetrahedron Lett. 2012, 53, 2887-2889. https://doi.org/10.1016/j.tetlet.2012.03.132

11. Han, X.-J. Tetrahedron Lett. 2007, 48, 2845-2849. https://doi.org/10.1016/j.tetlet.2007.02.101

12. Kim, S.; Kang, S.; Kim, G.; Lee, Y. J. Org. Chem. 2016, 81, 4048-4057. https://doi.org/10.1021/acs.joc.6b00341

13. Fadini, L. ; Togni, A. Chem. Commun. 2003, No. 1, 30-31. https://doi.org/10.1039/b210680a

14. Rajabi, F. ; Razavi, S. ; Luque, R. Green Chem. 2010, 12, 786-789. https://doi.org/10.1039/b926599f

15. Dai, L. ; Zhang, Y. ; Dou, Q. ; Wang, X. ; Chen, Y. Tetrahedron 2013, 69, 1712-1716. https://doi.org/10.1016/i.tet.2012.12.037 
16. Stevanovic, D. ; Pejović, A. ; Damljanović, I. ; Vukićević, M. ; Bogdanović, G. A. ; Vukićević, R. D. Tetrahedron Lett. 2012, 53, 6257-6260.

https://doi.org/10.1016/j.tetlet.2012.09.023

17. Du, X.-J. ; Wang, Z.-P. ; Hou, Y.-L. ; Zhang, C. ; Li, Z.-M. ; Zhao, W.-G. Tetrahedron Lett. 2014, 55, $1002-1005$. https://doi.org/10.1016/j.tetlet.2013.12.059

18. Lin, Y.-D. ; Kao, J.-Q. ; Chen, C.-T. Org. Lett. 2007, 9, 5195-5198.

https://doi.org/10.1021/ol702302y

19. For examples of other interesting protocols : Zaderenko, P. ; López, M. C. ; Ballesteros, P. J. Org. Chem. 1996, 61, 6825-6828.

https://doi.org/10.1021/jo960763y

20. Moran, J. ; Dorman, P. ; Beauchemin, A. M. Org. Lett. 2007, 9, 3893-3896.

https://doi.org/10.1021/ol701496b

21. Cravotto, G. ; Cintas, P. Chem. Soc. Rev. 2006, 35, 180-196.

https://doi.org/10.1039/B503848K

22. Mason, T. J. Chem. Soc. Rev. 1997, 26, 443-451.

https://doi.org/10.1039/cs9972600443

23. Zhdankin, V. V.; Stang, P. J. Chem. Rev. 2002, 102, 2523-2584.

https://doi.org/10.1021/cr010003+

24. Zhdankin, V. V.; Stang, P. J. Chem. Rev. 2008, 108, 5299-5358.

https://doi.org/10.1021/cr800332c

25. Yoshimura, A.; Zhdankin, V. V. Chem. Rev. 2016, 116, 3328-3435.

https://doi.org/10.1021/acs.chemrev.5b00547

26. Li, X.; Chen, P.; Liu, G. Beilstein J. Org. Chem. 2018, 14, 1813-1825.

https://doi.org/10.3762/bjoc.14.154

27. Flores, A.; Cots, E.; Berges, J.; Muñiz, K. Adv. Synth. Catal. 2019, 361, 2-25.

https://doi.org/10.1002/adsc.201800521

28. Dohi, T.; Takenaga, N.; Nakae, T.; Toyoda, Y.; Yamasaki, M.; Shiro, M.; Fujioka, H.; Maruyama, A.; Kita, Y. J. Am. Chem. Soc. 2013, 135, 4558-4566.

https://doi.org/10.1021/ja401074u

29. Levitre, G.; Dumoulin, A.; Retailleau, P.; Panossian, A.; Leroux, F. R.; Masson, G. J. Org. Chem. 2017, 82, 11877-11883.

https://doi.org/10.1021/acs.joc.7b01597

30. Boppisetti, J. K.; Birman, V. B. Org. Lett. 2009, 11, 1221-1223.

https://doi.org/10.1021/ol8029092

31. Banik, S. M.; Mennie, K. M.; Jacobsen, E. N. J. Am. Chem. Soc. 2017, 139, 9152-9155.

https://doi.org/10.1021/jacs.7b05160

32. Uyanik, M. ; Yasui, T. ; Ishihara, K. Angew. Chem. Int. Ed. 2013, 52, 9215-9218.

https://doi.org/10.1002/anie.201303559

33. Banik, S. M.; Medley, J. W.; Jacobsen, E. N. J. Am. Chem. Soc. 2016, 138, 5000-5003. https://doi.org/10.1021/jacs.6b02391

34. Banik, S. M. ; Medley, J. W. ; Jacobsen, E. N. J. Am. Chem. Soc. 2016, 138, 5000-5003. https://doi.org/10.1021/jacs.6b02391

35. Farid, U. ; Malmedy, F. ; Claveau, R. ; Albers, L. ; Wirth, T. Angew. Chem. Int. Ed. 2013, 52, 7018-7022. https://doi.org/10.1002/anie.201302358 
36. Wu, F.; Alom, N.-E.; Ariyarathna, J. P.; Naß, J.; Li, W. Angew. Chem. Int. Ed. 2019, 58, 11676-11680. https://doi.org/10.1002/anie.201904662

37. Alom, N.-E.; Kaur, N.; Wu, F.; Saluga, S. J.; Li, W. Chem. Eur. J. 2019, 25, 6902-6906. https://doi.org/10.1002/chem.201900549

38. Kaur, N.; Wu, F.; Alom, N.-E.; Ariyarathna, J. P.; Saluga S. J.; Li, W. Org. Biomol. Chem. 2019, $17,1643-1654$. https://doi.org/10.1039/C8OB02443J

39. Wu, F.; Stewart, S.; Ariyarathna, J. P.; Li, W. ACS Catal. 2018, 8, 1921-1925. https://doi.org/10.1021/acscatal.7b04060

40. Ariyarathna, J. P.; Wu, F.; Colombo, S. K.; Hillary, C. M.; Li, W. Org. Lett. 2018, 20, 6462-6466. https://doi.org/10.1021/acs.orglett.8b02771

41. Alom, N.-E.; Wu, F.; Li, W. Org. Lett. 2017, 19, 930-933. https://doi.org/10.1021/acs.orglett.7b00079

42. Alom, N.-E.; Rina, Y. A.; Li, W. Org. Lett. 2017, 19, 6204-6207. https://doi.org/10.1021/acs.orglett.7b03128

43. Wu, F.; Ariyarathna, J. P.; Alom, N.-E.; Kaur, N.; Li, W. Org. Lett. 2020, 22, 884-890. https://doi.org/10.1021/acs.orglett.9b04432

44. Wu, F.; Ariyarathna, J. P.; Kaur, N.; Alom, N.-E.; Kennell, M. L. ; Bassiouni, O. H. ; Li, W. Org. Lett. 2020, 22, 2135-2140.

https://doi.org/10.1021/acs.orglett.0c00081

45. Please see references 4-9.

46. Tao, J.; Murphy, G. K. Synthesis. 2019, 51, 3055-3059. https://doi.org/10.1055/s-0037-1611526

47. Zhao, Y.; Yim, W.-L.; Tan, C. K.; Yeung, Y.-Y. Org. Lett. 2011, 13, 4308-4311. https://doi.org/10.1021/ol2016466 